\title{
Biodiversity baseline of the French Guiana spider fauna
}

\author{
Vincent Vedel ${ }^{1,2^{*}}$, Christina Rheims ${ }^{3}$, Jérôme Murienne ${ }^{4}$ and Antonio Domingos Brescovit ${ }^{3}$
}

\begin{abstract}
The need for an updated list of spiders found in French Guiana rose recently due to many upcoming studies planned. In this paper, we list spiders from French Guiana from existing literature (with corrected nomenclature when necessary) and from 2142 spiders sampled in 12 sites for this baseline study. Three hundred and sixty four validated species names of spider were found in the literature and previous authors' works. Additional sampling, conducted for this study added another 89 identified species and 62 other species with only a genus name for now. The total species of spiders sampled in French Guiana is currently 515. Many other Morphospecies were found but not described as species yet. An accumulation curve was drawn with seven of the sampling sites and shows no plateau yet. Therefore, the number of species inhabiting French Guiana cannot yet be determined. As the very large number of singletons found in the collected materials suggests, the accumulation curve indicates nevertheless that more sampling is necessary to discover the many unknown spider species living in French Guiana, with a focus on specific periods (dry season and wet season) and on specific and poorly studied habitats such as canopy, inselberg and cambrouze (local bamboo monospecific forest).
\end{abstract}

Keywords: Araneae; Arachnids; Bio monitoring; French Guiana; Neotropics; Species richness

\section{Background}

Under the Streamline European Biodiversity Inventory 2010 protocols (SEBI) (Butchardt et al. 2010; Jones et al. 2011), species occurrences and abundances are currently only being assessed through survey of birds and butterflies. While there is a general agreement that those groups should continue to be monitored (EEA technical report No 11/2012) (EEA (2005), the Group on Earth Observations Biodiversity Observation Network (GEO BON), the European Biodiversity Observatory Network (EBON) (Reviewed in Scholes et al. 2008) and many authors specialized in this field (De Baan et al. 2012; Cardoso et al. 2011; Feest 2013; Feest et al. 2011) have recommended the survey and monitoring of additional groups to fill the taxonomic and ecological gaps.

Spiders have been identified as a meaningful additional indicator taxon by the European Commission FP7-

\footnotetext{
* Correspondence: vincent.vedel@ecofog.gf

'Laboratoire d'entomologie Entobios, 5 Bis rue François Thomas, 97310 Kourou, Guyane Française, France

Laboratoire d'écologie intégrative, UMR ECOFOG, Université des Antilles et de la Guyane, Campus Agronomique de Kourou, 97310 Kourou, French Guiana, France

Full list of author information is available at the end of the article
}

BioBio project (Targetti et al. 2012) not only because they represent well the local micro-fauna richness, but also because they are easy and cheap to sample, sensitive to changes (Cardoso et al. 2008), have little dispersal potential (New 1999), are abundant and diverse (Foelix 1996), represent differences in other species richness and diversity (Cardoso et al. 2008) and are recognized by stakeholders. Moreover, rigorous sampling protocols have just been set up (Cardoso 2009) and locally adapted (Vedel and Lalagüe 2013). They provide a complementary alternative to Lepidoptera in term of distribution and ecological functions as top predators of soil and lower vegetation communities (Cardoso et al. 2008) and are extremely diverse in tropical rain forest (Sørensen et al. 2002; Pinkus-Rendón et al. 2006; Coddington et al. 2009).

French Guiana is $97 \%$ covered by primary forest and hosts an exceptionally diverse and distinctive equatorial forest, part of the Amazonian tropical rainforest. This region also has an increasing demographic and economic development, which will raise conservation issues in the near future. As such it deserves special attention from the scientific community. The last integrative

\section{实}


spider species list for French Guiana is almost 70 years old (Di Caporiacco 1954) with few later additions (Drolshagen and Bäckstam 2011; Lopez 1988). In this study, our goal is to establish a baseline biodiversity reference for the spider fauna of French Guiana to enable further studies which will set spider monitoring as an efficient "tool" for assessment and monitoring local biodiversity.

\section{Results and discussion}

2142 spider specimens were sampled and sent for identification during this study. Identification results are summarized in Table 1. About 692 Morphospecies (M-S from hereonin) were singled out from this material with many M-S represented only by singletons. Many of these M-S could not be identified yet, and therefore are not included in the species list. In addition, many individuals could not be identified because they were either juveniles or undescribed, and they were therefore excluded from this list. Individuals identified only at the genus level are mainly species which are either not recognized yet, even with the sexual organs (often just one sex represented), or are a species new to science and therefore not described and not named yet. In any case they represent a species not found in the region until now.

After adding to Caporiacco (1954) list the sampling from this study and the previously identified materials from the two last authors, we obtained a total number of 515 species belonging to 45 families (Table 2). Therefore, 151 new species were added in this study and nine new families for French Guiana were also found: Amaurobiidae, Cyrtaucheniidae, Hersiliidae, Linyphiidae, Miturgidae, Oonopidae, Prodidomidae, Senoculidae and Synotaxidae (see Table 3 for the detailed list of species). From these 151 new species 89 species were named at the species level (Table 2), which indicates this study added 20\% more species names to the French Guianan total. Only 137 species described in Caporiacco's work (1954), which represent about $40 \%$ of the species number, were resampled in our study. This number is probably largely underestimated due to the lack of certain identification for many specimens.

The number of M-S found (692 M-S for 1617 spiders sampled) is similar to what was found in Bolivia and Peru; respectively 329 species out of 1109 specimens sampled and 635 species for 1821 specimens (reviewed

Table 1 Number of specimens collected for each location with the number of morpho-species recognized

\begin{tabular}{|c|c|c|c|c|c|c|}
\hline Sampling site & Coordinates & Season & $\begin{array}{c}\text { Number of } \\
\text { collected individuals }\end{array}$ & $\begin{array}{c}\text { Number of } \\
\text { morpho-species }\end{array}$ & $\begin{array}{l}\text { Type of } \\
\text { habitats }\end{array}$ & $\begin{array}{l}\text { Sampling } \\
\text { methods }\end{array}$ \\
\hline Crique Baggot & $\begin{array}{l}\text { 22N0329797- } \\
0501628\end{array}$ & Wet & 24 & 23 & $\mathrm{FF}$ & $B(1), S N(1), H(1)$ \\
\hline $\begin{array}{c}\text { Gentry plots (Petite Montagne Tortue, } \\
\text { Régina) }\end{array}$ & $\begin{array}{l}\text { 22N0362289- } \\
0477672\end{array}$ & $\begin{array}{l}\text { Wet, } \\
\text { Dry }\end{array}$ & 97 & 58 & WS, FF, TF & MT, WP \\
\hline Gentry plots (Laussat Ouest) & $\begin{array}{l}\text { 22N0213521- } \\
0605836\end{array}$ & $\begin{array}{l}\text { Wet, } \\
\text { Dry }\end{array}$ & 76 & 42 & WS, FF, TF & MT, WP \\
\hline Grand Connétable Island & $\begin{array}{l}\text { 22N0396505- } \\
0534312\end{array}$ & Wet & 15 & 10 & $\mathrm{Ol}$ & $\mathrm{H}(2)$ \\
\hline Kaw & $\begin{array}{l}\text { 22N0315898- } \\
0556000\end{array}$ & Wet & 78 & 60 & WF & $B(2), S N(2), S(2)$ \\
\hline La Trinité & $\begin{array}{l}\text { 22N0232748- } \\
0510994\end{array}$ & Dry & 439 & 242 & $F F, T F, I$ & $\begin{array}{c}B(6), S N(6), S(3) \\
\text { WP }\end{array}$ \\
\hline Mont Itoupé & - & Wet & 74 & 61 & I & WP, MT \\
\hline Nouragues & $\begin{array}{l}\text { 22N0314321- } \\
0446496\end{array}$ & Wet & 338 & 175 & $F F, T F, I$ & $\begin{array}{c}\mathrm{B}(6), \mathrm{SN}(6), \mathrm{S}(3) \\
\text { WP }\end{array}$ \\
\hline Nouragues & $\begin{array}{l}\text { 22N0307547- } \\
0450440\end{array}$ & Dry & 375 & 270 & $F F, T F, I$ & $\begin{array}{c}B(10), S N(10), S(5), \\
\text { WP }\end{array}$ \\
\hline Piste des compagnons & $\begin{array}{l}\text { 22N0310766- } \\
0564719\end{array}$ & Dry & 27 & 25 & TF & MT \\
\hline Saül & $\begin{array}{l}\text { 22N0253843- } \\
0400740\end{array}$ & Wet & 482 & 347 & $F F, T F, I$ & $B(6), S N-6), S(3)$, \\
\hline Savane-roche Virginie & $\begin{array}{l}\text { 22N0257866, } \\
\text { W0731672 }\end{array}$ & Dry & 117 & 43 & I & $\mathrm{SN}(2), \mathrm{H}(2)$ \\
\hline Total & & & 2142 & NA & & \\
\hline
\end{tabular}

Abbreviations: (FF Flooded Forest, TF Terra Firme, I Inselberg, WF Wet Forest, OI Oceanic Island, WS White Sand, $B$ Beating, SN Sweep Net, $S$ Sieve, $H$ by Hand, WP Window Pane trap, MT Malaise Trap. Numbers in brackets reflect the units of sampling effort for the active techniques applied. The wet season lasts from December until June and the dry season from July until November with some little variations. The total number of M-S cannot be determined (and it is therefore noted $N A=$ Not Applicable) because at some sites spiders were not photographed and could not be compared with other sites (those sites are the ones not used for the following analyses). 


\begin{tabular}{|c|c|c|}
\hline Sources & Species level & $\begin{array}{l}\text { M-S identified at the } \\
\text { Genus level }\end{array}$ \\
\hline $\begin{array}{l}\text { Former list published: } \\
\text { (Caporiacco 1954) }\end{array}$ & 364 & 0 \\
\hline $\begin{array}{l}\text { Brescovit's review } \\
\text { (Brescovit et al. 2011) }\end{array}$ & 34 & 2 \\
\hline Present study's samples & 55 & 60 \\
\hline Total & 453 & 62 \\
\hline
\end{tabular}

in Coddington et al. 2009), but represents ten times more than the number of spider species found in temperate forests (Coddington et al. 1996) and much more than found in Tanzania (170 species for 9096 specimens sampled) (Sørensen et al. 2002) and Malaysia (578 species for 6999 specimens collected) (Floren and Deeleman-Reinhold 2005) indicating the high diversity of the Amazonian areas. In Guyana, a neighbourhood country of French Guiana, only 351 species were found out of 5965 specimens collected (Coddington et al. 2009). Nonetheless, these comparisons have to be made with caution due to differences in sampling protocols including different techniques, efforts and the number of different habitats studied.

It is interesting to observe that the most diverse families are representative of most of the main feeding guilds of spiders (Dias et al. 2010; Cardoso et al. 2011): Salticidae with 153 species belong to the diurnal hunting spiders, the Araneidae with 113 species belong to the orb weavers, the Thomisidae with 39 identified species are representative of the ambush spiders, the Theridiidae with 33 species represent the entangled web weavers, the Corinnidae with 26 recognized species belong to the nocturnal hunting spiders and finally the Theraphosidae (the most diverse and numerous Mygalomorphae representative) with 17 species, ambush from their burrows. Nevertheless, the Ctenidae (15 species) and Trechaleidae (7 species) are probably much more diverse than we have found up until now. In this study, we collected about 60 different M-S belonging to the first family and 20 for the second.

The rate of Endemism is quite high, as noted by Caporiacco (1954) with 192 species out of 357 (53.8\%) never having been found outside French Guiana. In this study we cannot accurately evaluate endemism because of the bias made during the identification: most of the named species are fully identified because they were previously discovered and described from somewhere else, where spider studies were performed over a longer period of time. In any case, the number of endemic species has to be handled carefully here due to the poor comparison possible due to few studies achieved in the neighbouring regions (Venezuela, Surinam, Guyana and Amapá and Pará States in Brazil).

The accumulation curve shows a constant increase of the number of M-S (Table 4 and Figure 1) and does not reach a plateau, even on the calculated tendency curve. This plateau normally shows the total number of species present in a site or a region. Here, we cannot determine yet this number from the curve which indicates that we have to sample many more individuals to arrive at this plateau.

From the estimators, the minimum richness is evaluated at 1241 species by the Chao 1 estimator and the Jackknife estimator calculated $1680(+/-112)$ species. These results suggest we only know about one third (515 species identified out of around 1,500 species estimated) of the local spider fauna, which places French Guiana as a region of high diversity of spiders but also in a region where sampling and identification efforts have to be substantially increased to gain a sufficient knowledge in order to be able to use spider as a biodiversity assessment tool.

Now, although the aim of having a list of spiders to start with is reached, those results have to be taken as a first step in the arachnological biodiversity assessment of French Guiana and not as an exhaustive catalog of the spiders inhabiting the area. At each sampled site, although an inclusive sampling protocol was applied, the large number of singletons (between 50 to $70 \%$ of the individuals) indicates clearly that those sites are undersampled (Coddington et al. 2009). A sampling effort index comparing the ratios of abundance to species $(\mathrm{N} / \mathrm{S})$ (Colwell and Coddington 1994) gives a result of 54 (9096/170) for Tanzania, 12 for Malaysia, 17 for Guyana, 2.87 for Pérù and 3.37 for Bolivia. In our study, the ratio is $2.34(1617 / 692)$ which shows, in comparison to the other studies both that French Guianan sites are under-sampled and that the diversity is locally high; being comparable to Bolivia and Perù. Tanzania's study exhibits a high ratio because the sampling was intensive and also because the sites there were poorer in term of diversity.

Moreover, each site should be sampled at different times of the year as the wet and the dry seasons exhibit radical changes in environmental conditions which trigger a change in the communities of animals. In addition, some specific habitats such as canopies, inselbergs (granitic hill specific vegetation) or liana forests were not well sampled and might hold some unknown spider species too. Therefore, in order to assess the total species richness of the spiders of French Guiana, the sampling effort has to be substantially increased, in order that further quantitative studies applying more advanced methods to define the local fauna (Feest \& Cardoso 2012). Applying rigorous sampling protocols can be widely applied for local biodiversity assessment using spiders. 
Table 3 Current list of identified spiders from French Guiana with notes about the current names

\begin{tabular}{|c|c|c|c|}
\hline \multicolumn{4}{|c|}{ Guyane+espèces } \\
\hline Familyx & Species & New finding & Notes \\
\hline Amaurobiidae & Amaurobius brevis (Taczanowski, 1874) & B & incertae sedis/ Corinnidae \\
\hline Amaurobiidae & Amaurobius cayanus (Taczanowski, 1874) & B & incertae sedis/ Corinnidae \\
\hline Amaurobiidae & Amaurobius hirtus (Taczanowski, 1874) & B & incertae sedis/ Corinnidae \\
\hline Amaurobiidae & Amaurobius rufipes (Taczanowski, 1874) & B & incertae sedis/ Corinnidae \\
\hline Anyphaenidae & Aljassa n. sp.1 & V & \\
\hline Anyphaenidae & Anyphaenoides n. sp.1 & v & \\
\hline Anyphaenidae & Hibana melloleitaoi (Caporiacco, 1947) & V & \\
\hline Anyphaenidae & Katissa n. sp.1 & v & \\
\hline Anyphaenidae & Mesilla anyphaenoides (Caporiacco 1954) & & \\
\hline Anyphaenidae & Patrera armata (Chickering, 1940) & v & \\
\hline Anyphaenidae & Patrera n. sp.1 & v & \\
\hline Anyphaenidae & Patrera n. sp.2 & v & \\
\hline Anyphaenidae & Patrera n. sp.3 & v & \\
\hline Anyphaenidae & Patrera n. sp.4 & v & \\
\hline Anyphaenidae & Sillus furciger (Caporiacco 1954) & & \\
\hline Anyphaenidae & Wulfila n. sp.1 & V & \\
\hline Araneidae & Actinosoma pentacanthum (Walckenaer, 1841) & & \\
\hline Araneidae & Acacesia hamata (Hentz, 1847) & & \\
\hline Araneidae & Acacesia tenella (Koch, 1871) & & \\
\hline Araneidae & Alpaida deborae (Levi, 1988) & & \\
\hline Araneidae & Alpaida erythrothorax (Taczanowski, 1873) & & \\
\hline Araneidae & Alpaida graphica (Cambridge, 1889) & & \\
\hline Araneidae & Alpaida marmorata (Taczanowski, 1873) & & \\
\hline Araneidae & Alpaida sulphurea (Taczanowski, 1873) & & \\
\hline Araneidae & Alpaida truncata (Keyserling, 1865) & & \\
\hline Araneidae & Alpaida truncata obscura (Caporiacco 1954,1948) & & \\
\hline Araneidae & Alpaida truncata sexmaculata (Caporiacco 1954, 1948) & & \\
\hline Araneidae & Alpaida veniliae (Keyserling, 1865) & & \\
\hline Araneidae & Araneus appendiculatus (Taczanowski, 1873) & & \\
\hline Araneidae & Araneus contestationis (Caporiacco 1954) & & Nomem dubium \\
\hline Araneidae & Araneus decaspinus (Taczanowski, 1873) & & Nomem dubium \\
\hline Araneidae & Araneus guttatus (Keyserling, 1865) & & \\
\hline Araneidae & Araneus nigrocellatus (Caporiacco 1954) & & Nomen dubium \\
\hline Araneidae & Araneus venatrix (Koch, 1838) & & \\
\hline Araneidae & Argiope argentata (Fabricius, 1775) & & \\
\hline Araneidae & Argiope trifasciata (Forsskel, 1775) & & \\
\hline Araneidae & Cercidia n. sp.1 & & \\
\hline Araneidae & Chaetacis abrahami (Mello-Leitão, 1948) & B & \\
\hline Araneidae & Chaetacis aureola (Koch, 1836) & & \\
\hline Araneidae & Chaetacis cornuta (Taczanowski, 1873) & & \\
\hline Araneidae & Chaetacis necopinata (Chickering, 1960) & v & \\
\hline Araneidae & Cyclosa fililineata (Hingston, 1932) & V & \\
\hline Araneidae & Cyclosa nodosa (Cambridge, 1889) & & \\
\hline
\end{tabular}


Table 3 Current list of identified spiders from French Guiana with notes about the current names (Continued)

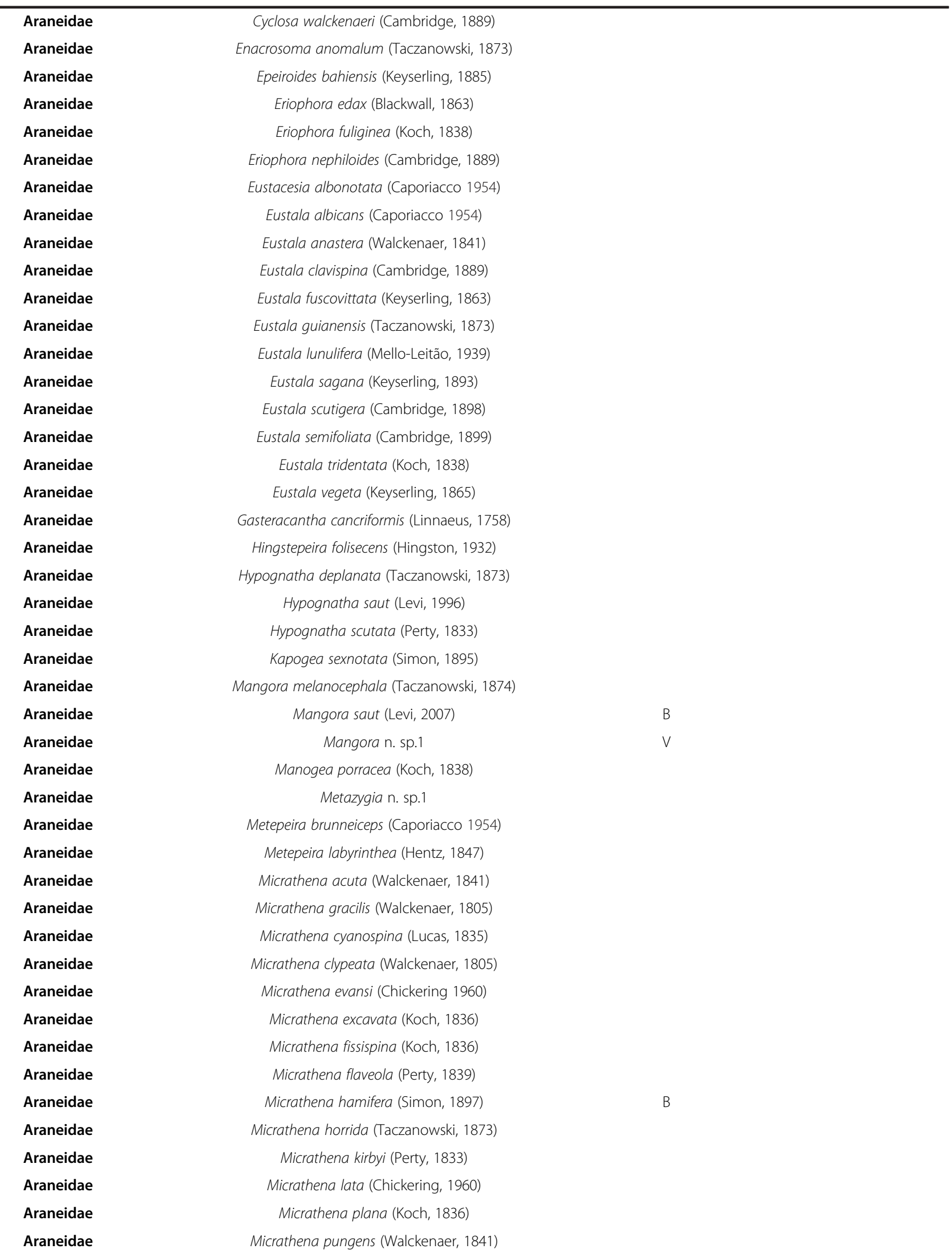


Table 3 Current list of identified spiders from French Guiana with notes about the current names (Continued)

\begin{tabular}{|c|c|c|c|}
\hline Araneidae & Micrathena saccata (Koch, 1836) & & \\
\hline Araneidae & Micrathena schreibersi (Perty 1833) & & \\
\hline Araneidae & Micrathena sexspinosa (Hahn, 1822) & & \\
\hline Araneidae & Micrathena spinosa (Linnaeus, 1758) & & \\
\hline Araneidae & Micrathena triangularis (Koch, 1836) & & \\
\hline Araneidae & Micrathena triangularispinosa (De Geer, 1778) & & \\
\hline Araneidae & Micrepeira hoeferi (Levi, 1995) & & \\
\hline Araneidae & Micrepeira tubulofaciens (Hingston, 1932) & & \\
\hline Araneidae & Neoscona benjamina (Walckenaer, 1841) & & Nomem dubium \\
\hline Araneidae & Neoscona nautica (Koch, 1875) & & \\
\hline Araneidae & Neoscona theisi (Walckenaer, 1841) & & \\
\hline Araneidae & Ocrepeira albopunctata (Taczanowski, 1879) & & \\
\hline Araneidae & Ocrepeira covillei (Levi, 1993) & & \\
\hline Araneidae & Ocrepeira n. sp.2 & & \\
\hline Araneidae & Parawixia audax (Blackwall, 1863) & & \\
\hline Araneidae & Parawixia kochi (Taczanowski, 1873) & & \\
\hline Araneidae & Parawixia velutina (Taczanowski, 1878) & & \\
\hline Araneidae & Scoloderus tuberculifer (Cambridge, 1889) & & \\
\hline Araneidae & Testudinaria quadripunctata (Taczanowski, 1879) & B & \\
\hline Araneidae & Verrucosa arenata (Walckenaer, 1841) & & \\
\hline Araneidae & Verrucosa septemmammata (Caporiacco 1954) & & \\
\hline Araneidae & Wagneriana jelskii (Taczanowski, 1873) & & \\
\hline Araneidae & Wagneriana tayos (Levi, 1991) & V & \\
\hline Araneidae & Wagneriana tauricornis (Cambridge, 1889) & & \\
\hline Araneidae & Wagneriana transitoria (Koch, 1839) & & \\
\hline Araneidae & Witica cayanus (Taczanowski, 1873) & & \\
\hline Araneidae & Wixia n. sp.1 & & \\
\hline Araneidae & Xylethrus n. sp.1 & & \\
\hline Barychelidae & Psalistops gasci (Maréchal, 1996) & & \\
\hline Caponiidae & Nops branicki (Taczanowski, 1874) & & \\
\hline Clubionidae & Elaver sericea (Cambridge, 1898) & & \\
\hline Clubionidae & Elaver n. sp.2 & V & \\
\hline Corinnidae & Apochinomma n. sp.1 & V & \\
\hline Corinnidae & Castianeira salticina (Taczanowski, 1874) & & \\
\hline Corinnidae & Castianeira n. sp.1 & V & \\
\hline Corinnidae & Castianeira n. sp.2 & V & \\
\hline Corinnidae & Corinna annulipes (Taczanowski, 1874) & & \\
\hline Corinnidae & Corinna anomala (Schmidt, 1971) & & \\
\hline Corinnidae & Corinna kochi (Petrunkevith, 1911) & V & \\
\hline Corinnidae & Corinna n. sp.4 & V & \\
\hline Corinnidae & Corinna n. sp.5 & V & \\
\hline Corinnidae & Corinna n. sp.6 & V & \\
\hline Corinnidae & Corinna n. sp.7 & V & \\
\hline Corinnidae & Medmassa septentrionalis (Caporiacco 1954) & & Nomem dubium \\
\hline Corinnidae & Methesis brevitarsa (Caporiacco 1954) & & \\
\hline
\end{tabular}


Table 3 Current list of identified spiders from French Guiana with notes about the current names (Continued)

\begin{tabular}{|c|c|c|c|}
\hline Corinnidae & Myrmecium n. sp.1 & V & \\
\hline Corinnidae & Myrmecium n. sp.2 & V & \\
\hline Corinnidae & Myrmecium n. sp.3 & V & \\
\hline Corinnidae & Myrmecium n. sp.4 & V & \\
\hline Corinnidae & Myrmecium bifasciatum (Taczanowski, 1874) & & \\
\hline Corinnidae & Myrmecotypus n. sp.1 & V & \\
\hline Corinnidae & Parachemmis hassleri (Gertsch, 1942) & V & \\
\hline Corinnidae & Parachemmis n. sp.1 & V & \\
\hline Corinnidae & Paradiestius n. sp.1 & V & \\
\hline Corinnidae & Tupirinna n. sp.1 & V & \\
\hline Corinnidae & Trachelas anomalus (Taczanowski, 1874) & & \\
\hline Corinnidae & Trachelas n. sp.1 & V & \\
\hline Corinnidae & Trachelas n. sp.2 & V & \\
\hline Corinnidae & Trachelas n. sp.3 & V & \\
\hline Ctenidae & Ancylometes bogotensis (Keyserling, 1876) & B & \\
\hline Ctenidae & Ancylometes rufus (Walckenaer, 1837) & & \\
\hline Ctenidae & Centroctenus auberti (Caporiacco 1954) & & \\
\hline Ctenidae & Ctenus ellacomei (Cambridge, 1902) & & \\
\hline Ctenidae & Ctenus n. sp.1 & V & \\
\hline Ctenidae & Ctenus n. sp.2 & V & \\
\hline Ctenidae & Ctenus n. sp.3 & & \\
\hline Ctenidae & Ctenus villasboasi (Mello-Leitao, 1949) & V & \\
\hline Ctenidae & Ctenus crulsi (Mello-Leitao, 1930) & V & \\
\hline Ctenidae & Ctenus dubius (Walckenaer, 1805) & & \\
\hline Ctenidae & Cupiennius bimaculatus (Taczanowski, 1874) & V & \\
\hline Ctenidae & Cupiennius foliatus (Cambridge, 1901) & & \\
\hline Ctenidae & Isoctenus latevittatus (Caporiacco 1954) & & Nomen nudum \\
\hline Ctenidae & Phoneutria fera (Perty, 1833) & & \\
\hline Ctenidae & Phoneutria reidyi (Cambridge, 1897) & & \\
\hline Cyrtaucheniidae & Fufius n. sp.1 & V & \\
\hline Deinopidae & Deinopis guianensis (Taczanowski, 1874) & & \\
\hline Dictynidae & Phantyna mandibularis (Taczanowski, 1874) & & \\
\hline Dipluridae & Harmonicon audeae (Maréchal \& Marty, 1998) & & \\
\hline Dipluridae & Harmonicon oiapoqueae (Drolshagen \& Bäckstam 2011) & & \\
\hline Dipluridae & Harmonicon rufescens (Cambridge, 1897) & & \\
\hline Dipluridae & Diplura nigra (Cambridge, 1897) & & \\
\hline Dipluridae & Ischnothele guianensis (Walkenaer, 1837) & & \\
\hline Dysderidae & Dysdera bicolor (Taczanowski, 1874) & & \\
\hline Eresidae & Eresus n. sp.1 & V & \\
\hline Eresidae & Eresus n. sp.2 & V & \\
\hline Filistatidae & Kukulcania hibernalis (Hentz, 1842) & & \\
\hline Gnaphosidae & Echemographis distincta (Caporiacco 1954) & & \\
\hline Hersiliidae & Neotama n. sp.1 & V & \\
\hline Hersiliidae & Ypypuera n. sp.1 & V & \\
\hline Idiopidae & Idiops opifex (Simon, 1889) & & \\
\hline
\end{tabular}


Table 3 Current list of identified spiders from French Guiana with notes about the current names (Continued)

\begin{tabular}{|c|c|c|c|}
\hline Linyphiidae & Ceratinopsis jelskii (Keyserling, 1886) & B & Nomem dubium \\
\hline Linyphiidae & Meioneta n. sp.1 & V & \\
\hline Lycosidae & Agalenocosa denisi (Caporiacco, 1947) & & \\
\hline Lycosidae & Aglaoctenus castaneus (Mello-Leitao, 1942) & V & \\
\hline Lycosidae & Aglaoctenus guianensis (Caporiacco 1954) & & Nomen dubium \\
\hline Lycosidae & Trochosa n. sp.1 & V & \\
\hline Lycosidae & Allocosa n. sp.1 & V & \\
\hline Lycosidae & Hogna vachoni (Caporiacco 1954) & & \\
\hline Lycosidae & Hogna ventrilineata (Caporiacco 1954) & & \\
\hline Lycosidae & Pardosa cayennensis (Taczanowski, 1874) & & \\
\hline Mimetidae & Ero n. sp.1 & V & \\
\hline Mimetidae & Ero n. sp.2 & V & \\
\hline Mimetidae & Gelanor mabelae (Chickering, 1947) & V & \\
\hline Mimetidae & Gelanor zonatus (Koch 1845) & & \\
\hline Miturgidae & Cheiracanthium inclusum (Hentz, 1847) & B & \\
\hline Miturgidae & Teminius insularis (Lucas, 1857) & B & \\
\hline Nephilidae & Nephila clavipes (Linnaeus, 1767) & & \\
\hline Nephilidae & Nephila cornuta (Pallas, 1772) & & \\
\hline Nephilidae & Nephilengys cruentata (Fabricius, 1775) & & \\
\hline Nesticidae & Nesticus citrinus (Taczanowski, 1874) & & \\
\hline Ochyroceratidae & Ochyrocera caeruleoamethystina (Lopez \& Lopez, 1997) & & \\
\hline Oonopidae & Neoxyphinus hispidus (Dumitresco \& Georgescu, 1987) & V & \\
\hline Oonopidae & Oonops n. sp.1 & V & \\
\hline Oxyopidae & Hamataliwa barroana (Keyserling 1887) & & \\
\hline Oxyopidae & Oxyopes haemorrhous (Mello-Leitao, 1929) & & \\
\hline Oxyopidae & Oxyopes maripae (Caporiacco 1954) & & \\
\hline Oxyopidae & Oxyopes masculinus (Caporiacco 1954) & & \\
\hline Oxyopidae & Oxyopes salticus (Hentz, 1845) & V & \\
\hline Oxyopidae & Peucetia macroglossa (Mello-Leitão, 1929) & & \\
\hline Oxyopidae & Tapinillus longipes (Taczanowski, 1872) & & \\
\hline Paratropididae & Paratropis papilligera (Cambridge, 1896) & & \\
\hline Philodromidae & Cleocnemis punctulata (Taczanowski, 1872) & & \\
\hline Philodromidae & Philodromus cayanus (Taczanowski, 1872) & & \\
\hline Pholcidae & Artema atlanta (Walckenaer, 1837) & & \\
\hline Pholcidae & Litoporus saul (Huber, 2000) & & \\
\hline Pholcidae & Mesabolivar cyaneus (Taczanowski, 1874) & & \\
\hline Pholcidae & Metagonia auberti (Caporiacco 1954) & & \\
\hline Pholcidae & Physocyclus globosus (Taczanowski, 1874) & B & \\
\hline Pholcidae & Smeringopus pallidus (Blackwall, 1858) & B & \\
\hline Pisauridae & Architis spinipes (Taczanowski, 1874) & & \\
\hline Pisauridae & Architis tenuis (Simon, 1898) & B & \\
\hline Pisauridae & Dolomedes elegans (Taczanowski, 1874) & & \\
\hline Pisauridae & Thaumasia benoisti (Caporiacco 1954) & & \\
\hline Pisauridae & Thaumasia marginella (Koch, 1847) & & \\
\hline Pisauridae & Tinus nigrinus (Cambridge, 1901) & & \\
\hline
\end{tabular}


Table 3 Current list of identified spiders from French Guiana with notes about the current names (Continued)

\begin{tabular}{|c|c|c|c|}
\hline Prodidomidae & Zimiris doriai (Simon, 1882) & B & \\
\hline Salticidae & Acragas flavescens (Caporiacco 1954) & & Nomen dubium \\
\hline Salticidae & Albionella guianensis (Caporiacco 1954) & V & \\
\hline Salticidae & Alcmena trifasciata (Caporiacco 1954) & & Nomen dubium \\
\hline Salticidae & Amycus ectipus (Simon, 1900) & V & \\
\hline Salticidae & Amycus effeminatus (Caporiacco 1954) & V & \\
\hline Salticidae & Amycus favicomis (Simon, 1900) & V & \\
\hline Salticidae & Amycus patellaris (Caporiacco 1954) & & Nomen dubium \\
\hline Salticidae & Anasaitis canosa (Walckenaer, 1837) & & \\
\hline Salticidae & Beata rustica (Peckham \& Peckham, 1895) & & \\
\hline Salticidae & Breda apicalis (Simon, 1901) & V & \\
\hline Salticidae & Capidava variegata (Caporiacco 1954) & & Nomen dubium \\
\hline Salticidae & Chinoscopus gracilis (Taczanowski, 1872) & & \\
\hline Salticidae & Chinoscopus maculipes (Crane, 1943) & & \\
\hline Salticidae & Chira guianensis (Taczanowski, 1871) & & \\
\hline Salticidae & Chira simoni (Galiano, 1961) & V & \\
\hline Salticidae & Chira spinipes (Taczanowski, 1871) & & \\
\hline Salticidae & Chira thysbe (Simon, 1902) & V & \\
\hline Salticidae & Chira trivittata (Taczanowski, 1871) & & \\
\hline Salticidae & Chirothecia crassipes (Taczanowski, 1878) & & \\
\hline Salticidae & Chloridusa n. sp.1 & V & \\
\hline Salticidae & Chloridusa n. sp.2 & V & \\
\hline Salticidae & Cobanus n. sp.1 & V & \\
\hline Salticidae & Corythalia heliophanina (Taczanowski, 1871) & & \\
\hline Salticidae & Corythalia luctuosa (Caporiacco 1954) & & \\
\hline Salticidae & Corythalia tropica (Mello-Leitão, 1939) & & \\
\hline Salticidae & Corythalia variegata (Caporiacco 1954) & & Nomen dubium \\
\hline Salticidae & Corythalia walecki (Taczanowski, 1871) & & \\
\hline Salticidae & Cyllodania fasciata (Caporiacco 1954) & V & \\
\hline Salticidae & Cyllistella n. sp.1 & V & \\
\hline Salticidae & Dendryphantes coccineocinctus (Caporiacco 1954) & & Nomen dubium \\
\hline Salticidae & Dendryphantes spinosissimus (Caporiacco 1954) & & Nomen dubium \\
\hline Salticidae & Euophrys ambigua (Koch, 1846) & & \\
\hline Salticidae & Eustiromastix bahiensis (Galiano, 1979) & V & \\
\hline Salticidae & Eustiromastix guianae (Caporiacco 1954) & & \\
\hline Salticidae & Eustiromastix major (Simon, 1902) & & \\
\hline Salticidae & Fluda n. sp.1 & V & \\
\hline Salticidae & Freya decorata (Koch, 1846) & & \\
\hline Salticidae & Freya disparipes (Caporiacco 1954) & & \\
\hline Salticidae & Freya grisea (Cambridge, 1901) & V & \\
\hline Salticidae & Freya perelegans (Simon, 1902) & V & \\
\hline Salticidae & Frigga coronigera (Koch, 1846) & V & \\
\hline Salticidae & Frigga kessleri (Taczanowski, 1872) & & \\
\hline Salticidae & Habronattus paratus (Peckham \& Peckham, 1896) & V & \\
\hline Salticidae & Helvetia cancrimana (Taczanowski, 1872) & & \\
\hline
\end{tabular}


Table 3 Current list of identified spiders from French Guiana with notes about the current names (Continued)

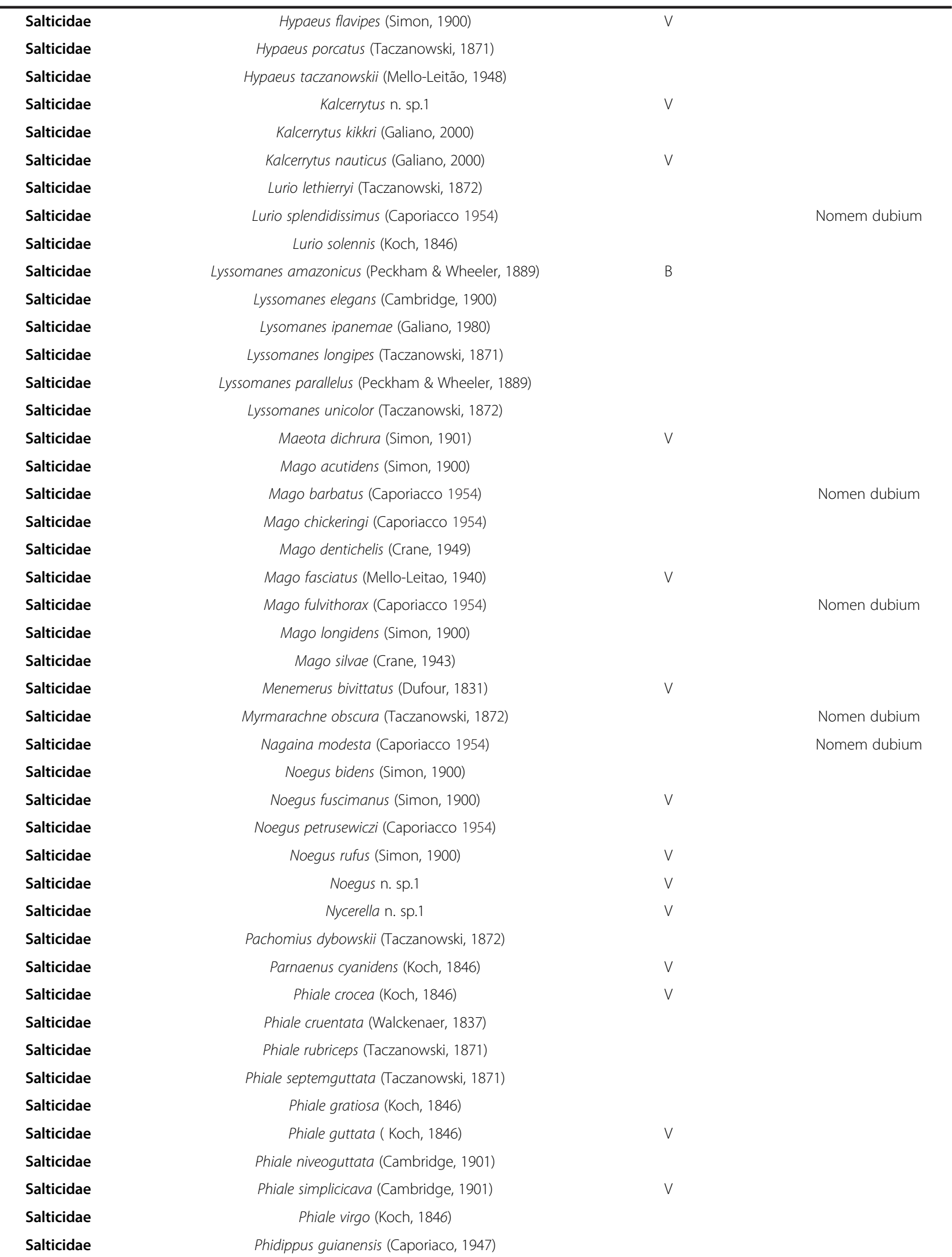


Table 3 Current list of identified spiders from French Guiana with notes about the current names (Continued)

\begin{tabular}{|c|c|c|c|}
\hline Salticidae & Phidippus triangulifer (Caporiacco 1954) & & Nomen dubium \\
\hline Salticidae & Platycryptus magnus (Peckham \& Peckham, 1894) & V & \\
\hline Salticidae & Plexippus paykulli (Audouin, 1826) & V & \\
\hline Salticidae & Psecas bubo (Taczanowski, 1871) & & \\
\hline Salticidae & Pseudopartona ornata (Caporiacco 1954) & & \\
\hline Salticidae & Rhene jelskii (Taczanowski, 1871) & & \\
\hline Salticidae & Romitia n. sp.1 & V & \\
\hline Salticidae & Rudra wagae (Taczanowski, 1872) & & \\
\hline Salticidae & Salticus albosignatus (Taczanowski, 1849) & & Nomen dubium \\
\hline Salticidae & Salticus bidens (Taczanowski, 1872) & & Nomen dubium \\
\hline Salticidae & Salticus cabanisi (Taczanowski, 1872) & & Nomen dubium \\
\hline Salticidae & Salticus cayanus (Taczanowski, 1871) & & Nomem dubium \\
\hline Salticidae & Salticus crassipes (Taczanowski, 1871) & & Nomen dubium \\
\hline Salticidae & Salticus cylindricus (Walckenaer, 1837) & & Nomem dubium \\
\hline Salticidae & Salticus deplanatus (Taczanowski, 1871) & & Nomem dubium \\
\hline Salticidae & Salticus dryocopinus (Taczanowski, 1871) & & Nomem dubium \\
\hline Salticidae & Salticus elaterinus (Taczanowski, 1871) & & Nomem dubium \\
\hline Salticidae & Salticus emaciatus (Walckenaer, 1837) & & Nomem dubium \\
\hline Salticidae & Salticus fulvatus (Fabricius, 1896) & & \\
\hline Salticidae & Salticus hamatinus (Taczanowski, 1849) & & Nomem dubium \\
\hline Salticidae & Salticus longimanus (Taczanowski, 1871) & & Nomem dubium \\
\hline Salticidae & Salticus mandibularis (Taczanowski, 1871) & & Nomem dubium \\
\hline Salticidae & Salticus marmottani (Taczanowski, 1871) & & Nomem dubium \\
\hline Salticidae & Salticus maronicus (Taczanowski, 1871) & & Nomem dubium \\
\hline Salticidae & Salticus miniaceus (Taczanowski, 1871) & & Nomem dubium \\
\hline Salticidae & Salticus minutus (Taczanowski, 1871) & & Nomem dubium \\
\hline Salticidae & Salticus nigerrimus (Taczanowski, 1871) & & Nomem dubium \\
\hline Salticidae & Salticus olivacens (Taczanowski, 1871) & & Nomem dubium \\
\hline Salticidae & Salticus ornatus (Taczanowski, 1871) & & Nomem dubium \\
\hline Salticidae & Salticus paederinus (Taczanowski, 1871) & & Nomem dubium \\
\hline Salticidae & Salticus platycephalus (Taczanowski, 1871) & & Nomem dubium \\
\hline Salticidae & Salticus quadriguttatus (Taczanowski, 1871) & & Nomem dubium \\
\hline Salticidae & Salticus radoszkowskii (Taczanowski, 1871) & & Nomem dubium \\
\hline Salticidae & Salticus rubescens (Walckenaer, 1837) & & Nomem dubium \\
\hline Salticidae & Salticus ruficeps (Taczanowski, 1871) & & Nomem dubium \\
\hline Salticidae & Salticus salutanus (Taczanowski, 1871) & & Nomem dubium \\
\hline Salticidae & Salticus sericeus (Taczanowski, 1871) & & Nomem dubium \\
\hline Salticidae & Salticus sexfasciatus (Taczanowski, 1871) & & Nomem dubium \\
\hline Salticidae & Salticus simoni (Taczanowski, 1871) & & Nomem dubium \\
\hline Salticidae & Salticus solskii (Taczanowski, 1871) & & Nomem dubium \\
\hline Salticidae & Salticus superciliatus (Walckenaer, 1837) & & Nomem dubium \\
\hline Salticidae & Salticus tenebrosus (Walckenaer, 1837) & & Nomem dubium \\
\hline Salticidae & Salticus tenuis (Taczanowski, 1871) & & Nomem dubium \\
\hline Salticidae & Salticus trematus (Walckenaer, 1837) & & Nomem dubium \\
\hline Salticidae & Salticus tricinctus (Taczanowski, 1871) & & Nomem dubium \\
\hline
\end{tabular}


Table 3 Current list of identified spiders from French Guiana with notes about the current names (Continued)

\begin{tabular}{|c|c|c|c|}
\hline Salticidae & Salticus uassanus (Taczanowski, 1871) & & Nomem dubium \\
\hline Salticidae & Salticus verrauxi (Taczanowski, 1871) & & Nomem dubium \\
\hline Salticidae & Sarinda atrata (Taczanowski, 1871) & & \\
\hline Salticidae & Sarinda cayennensis (Taczanowski, 1871) & & \\
\hline Salticidae & Sarinda longula (Taczanowski, 1871) & & \\
\hline Salticidae & Scopocira melanops (Taczanowski, 1871) & & \\
\hline Salticidae & Siloca septentrionalis (Caporiacco 1954) & & \\
\hline Salticidae & Soesilarishius n. sp.1 & V & \\
\hline Salticidae & Soesilarishius n. sp.2 & V & \\
\hline Salticidae & Synemosyna myrmeciaeformis (Taczanowski, 1871) & & \\
\hline Salticidae & Synemosyna subtilis (Taczanowski, 1871) & & Nomem dubium \\
\hline Salticidae & Synemosyna lucasi (Taczanowski, 1871) & & \\
\hline Salticidae & Thiodina branicki (Taczanowski, 1871) & & \\
\hline Salticidae & Thiodina melanogaster (Mello-Leitão, 1917) & V & \\
\hline Salticidae & Thiodina pallida (Koch, 1846) & & \\
\hline Salticidae & Tutelina iridea (Caporiacco 1954) & & Nomem dubium \\
\hline Salticidae & Viciria chabanaudi (Fage, 1923) & & \\
\hline Salticidae & Wedoquella n. sp.1 & & \\
\hline Salticidae & Zuniga magna (Peckham \& Peckham, 1892) & B & \\
\hline Scytodidae & Scytodes fusca (Walckenaer, 1837) & & \\
\hline Scytodidae & Scytodes lineatipes (Taczanowski, 1874) & & \\
\hline Scytodidae & Scytodes longipes (Lucas, 1844) & & \\
\hline Senoculidae & Senoculus canaliculatus (Cambridge, 1902) & B & \\
\hline Senoculidae & Senoculus maronicus (Taczanowski, 1872) & & \\
\hline Sparassidae & Guadana n. sp.1 & V & \\
\hline Sparassidae & Olios cayanus (Taczanowski, 1872) & & \\
\hline Sparassidae & Olios nigriventris (Taczanowski, 1872) & & \\
\hline Sparassidae & Olios quinquelineatus (Taczanowski, 1872) & & \\
\hline Sparassidae & Olios roeweri (Caporiacco 1954) & & \\
\hline Sparassidae & Olios rubripes (Taczanowski, 1872) & & \\
\hline Sparassidae & Olios velox (Simon, 1880) & V & \\
\hline Sparassidae & Polybetes pythagoricus (Holmberg, 1875) & & \\
\hline Sparassidae & Pseudosparianthis megalopalpa (Caporiacco 1954) & & \\
\hline Sparassidae & Sampaiosia crulsi (Mello-Leitão, 1930) & V & \\
\hline Sparassidae & Sparianthina rufescens (Mello-Leitão, 1940) & V & \\
\hline Sparassidae & Sparianthis amazonica (Simon, 1880) & V & \\
\hline Sparassidae & Thomasettia n. sp.1 & V & \\
\hline Sparassidae & Vindullus gracilipes (Taczanowski, 1872) & & \\
\hline Synotaxidae & Synotaxus n. sp.1 & V & \\
\hline Tetragnathidae & Azilia vachoni (Caporiacco 1954) & & \\
\hline Tetragnathidae & Chrysometa minuta (Keyserling, 1883) & & \\
\hline Tetragnathidae & Leucauge acuminata (Cambridge, 1889) & & \\
\hline Tetragnathidae & Leucauge argyra (Walckenaer, 1849) & & \\
\hline Tetragnathidae & Leucauge branickii (Taczanowski, 1874) & & \\
\hline Tetragnathidae & Leucauge funebris (Mello-Leitão, 1930) & & \\
\hline
\end{tabular}


Table 3 Current list of identified spiders from French Guiana with notes about the current names (Continued)

\begin{tabular}{|c|c|c|c|}
\hline Tetragnathidae & Leucauge pulcherrima (Keyserling, 1865) & & \\
\hline Tetragnathidae & Leucauge saphes (Chamberlain \& Ivie, 1936) & & \\
\hline Tetragnathidae & Leucauge taczanowskii (Marx, 1893) & & \\
\hline Tetragnathidae & Leucauge venusta (Walckenaer, 1841) & & \\
\hline Tetragnathidae & Metabus ocellatus (Keyserling, 1864) & B & \\
\hline Tetragnathidae & Opas caudacuta (Taczanowski, 1873) & & \\
\hline Tetragnathidae & Opas lugens, (Cambridge 1896) & & \\
\hline Tetragnathidae & Tetragnatha filiformata (Roewer, 1942) & & \\
\hline Tetragnathidae & Tetragnatha gibbula (Roewer, 1942) & & \\
\hline Theraphosidae & Acanthopelma beccarii (Caporiacco 1954) & & \\
\hline Theraphosidae & Acanthoscurria simoensi (Vol, 2000) & & \\
\hline Theraphosidae & Avicularia avicularia (Linnaeus, 1758) & & \\
\hline Theraphosidae & Avicularia avicularia variegata (Cambridge, 1896) & & \\
\hline Theraphosidae & Avicularia metallica (Ausserer, 1875) & & \\
\hline Theraphosidae & Avicularia holmbergi (Thorell, 1890) & & \\
\hline Theraphosidae & Avicularia lycosiformis (Koch, 1846) & & Nomen dubium \\
\hline Theraphosidae & Avicularia surinamensis (Strand, 1907) & & \\
\hline Theraphosidae & Ephebopus cyanognathus (West \& Marshall, 2000) & & \\
\hline Theraphosidae & Ephebopus murinus (Walckenaer, 1837) & & \\
\hline Theraphosidae & Ephebopus rufescens (West \& Marshall, 2000) & & \\
\hline Theraphosidae & Hapalopus guianensis (Caporiacco 1954) & & \\
\hline Theraphosidae & Magulla Janeira (Keyserling, 1891) & & \\
\hline Theraphosidae & Neostenotarsus (Tesmoingt \& Schmidt, 2002) & & \\
\hline Theraphosidae & Tapinauchenius gigas (Caporiacco 1954) & & \\
\hline Theraphosidae & Tapinauchenius violaceus (Mello-Leitão, 1930) & & \\
\hline Theraphosidae & Teraphosa blondi (Latreille, 1804) & & \\
\hline Theraphosidae & Vitalius vellutinus (Mello-Leitao, 1923) & & \\
\hline Theridiidae & Achaearanea hieroglyphica (Mello-Leitão, 1940) & & \\
\hline Theridiidae & Anelosimus chickeringi (Levi, 1956) & B & \\
\hline Theridiidae & Anelosimus eximius (Keyserling, 1884) & B & \\
\hline Theridiidae & Anelosimus jucundus (Cambridge, 1896) & B & \\
\hline Theridiidae & Anelosimus nigrescens (Keyserling 1884) & B & \\
\hline Theridiidae & Anelosimus rupununi (Levi, 1956) & B & \\
\hline Theridiidae & Anelosimus studiosus (Hentz, 1850) & B & \\
\hline Theridiidae & Argyrodes benedicti (Lopez 1988) & & \\
\hline Theridiidae & Argyrodes coactatus (Lopez 1988) & & \\
\hline Theridiidae & Argyrodes elevatus (Taczanowski, 1873) & & \\
\hline Theridiidae & Argyrodes nephilae (Taczanowski, 1873) & & \\
\hline Theridiidae & Chrysso albomaculata (Cambridge, 1882) & & \\
\hline Theridiidae & Chrysso pulcherrima (Mello-Leitão, 1917) & B & \\
\hline Theridiidae & Coleosoma acutiventer (Keyserling, 1884) & B & \\
\hline Theridiidae & Cryptachaea hirta (Taczanowski, 1873) & & \\
\hline Theridiidae & Cryptachaea migrans (Keyserling, 1884) & & \\
\hline Theridiidae & Cryptachaea pusillana (Roewer, 1942) & & \\
\hline Theridiidae & Cryptachaea rostrata (Cambridge, 1864) & & \\
\hline
\end{tabular}


Table 3 Current list of identified spiders from French Guiana with notes about the current names (Continued)

\begin{tabular}{|c|c|c|c|}
\hline Theridiidae & Dipoena n. sp.1 & V & \\
\hline Theridiidae & Dipoena n. sp.2 & V & \\
\hline Theridiidae & Episinus n. sp.1 & & \\
\hline Theridiidae & Faiditus americanus (Taczanowski, 1874) & & \\
\hline Theridiidae & Faiditus caudatus (Taczanowski, 1874) & & \\
\hline Theridiidae & Faiditus dracus (Chamberlin \& Ivie, 1936) & B & \\
\hline Theridiidae & Faiditus globosus (Keyserling, 1884) & B & \\
\hline Theridiidae & Neospintharus triangularis (Taczanowski, 1873) & B & \\
\hline Theridiidae & Parasteatoda tepidariorum (Koch, 1841) & B & \\
\hline Theridiidae & Rhomphaea paradoxa (Taczanowski, 1896) & B & \\
\hline Theridiidae & Steatoda ancorata (Holmberg, 1876) & B & \\
\hline Theridiidae & Nesticodes rufipes (Lucas, 1846) & & \\
\hline Theridiidae & Theridion incertissimum (Caporiacco 1954) & & \\
\hline Theridiidae & Theridion rubrolineatum (Taczanowski, 1874) & & Nomem dubium \\
\hline Theridiidae & Theridula gonygaster (Simon, 1873) & B & \\
\hline Theridiosomatidae & Naatlo splendida (Taczanowski, 1879) & B & \\
\hline Theridiosomatidae & Plato juberthiei (Lopez, 1996) & & \\
\hline Thomisidae & Acentroscelus guianensis (Taczanowski, 1872) & & \\
\hline Thomisidae & Acentroscelus nigrianus (Mello-Leitão, 1929) & & \\
\hline Thomisidae & Acentroscelus n. sp.1 & V & \\
\hline Thomisidae & Bucranium taurifrons (Cambridge, 1881) & & \\
\hline Thomisidae & Bucranium n. sp.1 & V & \\
\hline Thomisidae & Diaea n. sp.1 & V & \\
\hline Thomisidae & Epicadus heterogaster (Guérin, 1829) & & \\
\hline Thomisidae & Epicadinus trispinosus (Taczanowski, 1872) & & \\
\hline Thomisidae & Erissus truncatifrons (Simon, 1895) & & \\
\hline Thomisidae & Misumena citreoides (Taczanowski, 1872) & & \\
\hline Thomisidae & Misumena maronica (Caporiacco 1954) & & \\
\hline Thomisidae & Misumena nigripes (Taczanowski, 1872) & & \\
\hline Thomisidae & Misumenops guianensis (Taczanowski, 1872) & & \\
\hline Thomisidae & Monaeses lucasi (Taczanowski, 1872) & V & \\
\hline Thomisidae & Onoculus echinatus (Taczanowski, 1872) & & \\
\hline Thomisidae & Onoculus pentagonus (Keyserling, 1880) & V & \\
\hline Thomisidae & Platyarachne episcopalis (Taczanowski, 1872) & & \\
\hline Thomisidae & Runcinioides argenteus (Mello-Leitão, 1929) & & \\
\hline Thomisidae & Stephanopis quinquetuberculata (Taczanowski, 1872) & & \\
\hline Thomisidae & Strophius n. sp.1 & V & \\
\hline Thomisidae & Synema aequinoctiale (Taczanowski, 1872) & & \\
\hline Thomisidae & Synema bipunctatum (Taczanowski, 1872) & & \\
\hline Thomisidae & Synema bishopi (Caporiacco 1954) & & \\
\hline Thomisidae & Synema maculatovittatum (Caporiacco 1954) & & \\
\hline Thomisidae & Tmarus candefactus (Caporiacco 1954) & & \\
\hline Thomisidae & Tmarus geayi (Caporiacco 1954) & & \\
\hline Thomisidae & Tmarus grandis (Mello-Leitão, 1929) & & \\
\hline Thomisidae & Tmarus hystrix (Caporiacco 1954) & & \\
\hline
\end{tabular}




\section{Table 3 Current list of identified spiders from French Guiana with notes about the current names (Continued)}

\begin{tabular}{|c|c|c|c|}
\hline Thomisidae & Tmarus intentus (Cambridge, 1892) & & \\
\hline Thomisidae & Tmarus jelskii (Taczanowski, 1872) & & \\
\hline Thomisidae & Tmarus littoralis (Keyserling, 1880) & & \\
\hline Thomisidae & Tmarus obesus (Mello-Leitão, 1929) & & \\
\hline Thomisidae & Tobias albovittatus (Caporiacco 1954) & & \\
\hline Thomisidae & Tobias corticatus (Mello-Leitão, 1917) & & \\
\hline Thomisidae & Tobias cornutus (Taczanowski, 1872) & & \\
\hline Thomisidae & Tobias taczanowskii (Roewer, 1951) & V & \\
\hline Thomisidae & Tobias trituberculatus (Taczanowski, 1872) & & \\
\hline Thomisidae & Uraarachne vittata (Caporiacco 1954) & & \\
\hline Titanoecidae & Goeldia patellaris (Simon, 1892) & & \\
\hline Trechaleidae & Enna jullieni (Simon \& Duss, 1898) & & \\
\hline Trechaleidae & Paradossenus longipes (Taczanowski, 1874) & & \\
\hline Trechaleidae & Rhoicinus n. sp.1 & V & \\
\hline Trechaleidae & Syntrechalea reimoseri (Caporiacco, 1947) & V & \\
\hline Trechaleidae & Trechalea n. sp.1 & V & \\
\hline Trechaleidae & Trechalea n. sp.2 & V & \\
\hline Trechaleidae & Trechalea n. sp.3 & V & \\
\hline Uloboridae & Miagrammopes n. sp.1 & V & \\
\hline Uloboridae & Philiponnella semiplumosa (Simon, 1893) & & \\
\hline Uloboridae & Zosis geniculata (Olivier, 1789) & & \\
\hline Zodariidae & Tristichops coerulescens (Taczanowski, 1874) & & Nomem dubium \\
\hline
\end{tabular}

The numbers assigned to the M-S indicate only the order they were examined. B means that this species was not taken into consideration by the former list but was found published in the literature by Brescovit et al. 2011 and therefore added. V means that this species is new for French Guiana and found during the sampling expeditions organized for this study.

\section{Conclusions}

This study sets a starting point for the spider richness described and expected for French Guiana, in order to be able to use spiders as a "bioindication tool", as recommended by several commissions and organizations, for future biodiversity assessments.

After a literature review and several sampling excursions, the total number of spider species found in French Guiana is now at 515. This revision added 151 new species and nine new families for this region, which make a more credible start for upcoming spider studies. Many other M-S were found but not described as species yet. This study shows that few collections in Guianese forestry habitats brought many new species to the list for French Guiana, which suggests both that the area holds a high diversity of spiders and that this diversity was poorly explored.

The accumulation curve does not yet show the maximum of species number inhabiting this equatorial region but the species richness indices shows the tropical

Table 4 Sampling sites used in the accumulation curve

\begin{tabular}{cccc}
\hline Sites & Number of individuals & Number of M-S & Number of « new » M-S in each site \\
\hline Nouragues (dry season) & 375 & 270 & 270 \\
Crique Baggot & 24 & 23 & 11 \\
La Trinité & 439 & 242 & 132 \\
Gentry plots (Laussat Ouest & 76 & 42 & 25 \\
Gentry plots (Petite Montagne Tortue, Régina) & 97 & 58 & 52 \\
Saül & 482 & 347 & 164 \\
Savane roche Virginie & 124 & 50 & 38 \\
Total & 1617 & & 692
\end{tabular}

Sites were written in the chronological order they were sampled. The fourth column indicates the number of M-S not found in the previous sampled sites, starting from the top. Therefore they are considered as "new" for the sampling. 


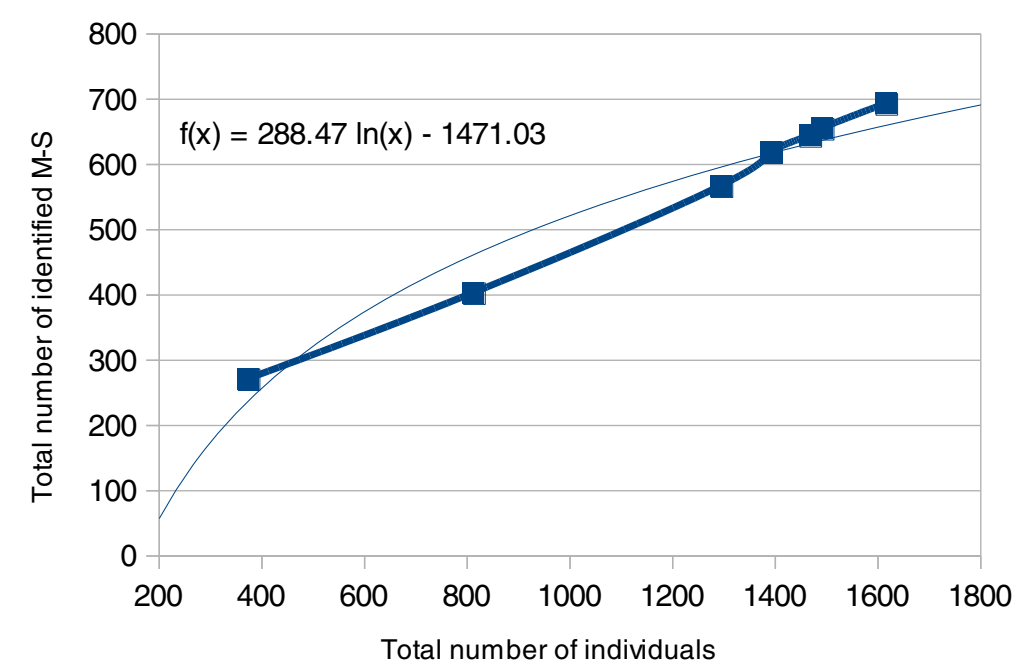

Figure 1 Accumulation curve showing the slope of the increase of $\mathbf{M}-\mathrm{S}$ numbers by the number of specimens collected. The tendency curve (light blue) was added, including its equation.

rainforest of French Guiana would host between 1241 and 1792 spider species. They also indicate that more sampling is necessary to complement our current knowledge in this regard. Finally, we can speculate that sampling specific periods (dry season and wet season) and specific and poorly studied habitats such as canopy, inselberg, cambrouze would bring many unknown spider species.

\section{Methods}

\section{Published data compilation}

The official National Natural Patrimony Inventory (INPN: http://inpn.mnhn.fr) currently lists 138 Araneae species for French Guiana. This list was checked and augmented by a complete survey of the literature from a database of Neotropical spiders (Brescovit et al. 2011). Species names were checked with reference to the world catalog of spiders 13.0 (Platnick 2012) and Prószyński's (2012) catalog of world.

\section{Study sites}

Sampling was performed by the authors in 12 sites of forest in French Guiana (Figure 2) at different periods during 3 years (2009-2011), to complement the number of species found in the existing literature. The name of the sites are given to the forest area were samples were collected. All of these sites are undisturbed forestry habitats. These sites were chosen for insect collecting expeditions organized by INRA (Institut National de la Recherche Agronomique) (the two Gentry sites), by the CNRS (Kaw) by the first author (Crique Baggot, Savane Roche Virginie, Piste des compagnons), and the remainder by the SEAG (Société Entomologique des Antilles et de la Guyane) for all the other sites.
The sites are located in different part of French Guiana covering almost all the region except the extreme southeast and the west (Figure 2), where most samples of Caporiacco's study were conducted (Caporiacco 1954). At each site several habitats (such as river side, swamps and terra firme) were sampled (Table 1 for details), offering altogether a good geographical coverage of the spiders found in French Guiana.

\section{Sampling protocol}

In order to collect the maximum number of spiders from every ecological stratum of each habitat, different active techniques and innovative methods such as traps were used (Vedel et al. 2011). Active techniques consist of sampling spiders using a sieve for filtering the soil and the leaf litter, a triangular sweep net for catching spiders inhabiting the low vegetation from $10 \mathrm{~cm}$ to $1.5 \mathrm{~m}$, a beating tray to collect individuals living from $1.5 \mathrm{~m}$ to $2.5 \mathrm{~m}$ in the higher vegetation and by hand to collect visible spiders on trunk or on silk webs. The innovative methods were Malaise traps and window pane traps, normally used to catch insects, which were recognized as efficient at collecting spiders (Vedel et al. 2011). Because of the heterogeneity of the sampling and the initial aim of the study, which was only to increase the number of spider species found, rigorous standardized protocol (Vedel and Lalagüe 2013) was applied only at only the sites Nouragues (wet season), Nouragues (dry season), Crique Baggot, La Trinité, Saül and Savane Roche Virginie. The sampling effort is an equal mix of the active techniques described above where one unit represents one hour of an active sampling technique. For the two Gentry plots spiders were collected only by traps (six window pane traps and six Malaise traps on 


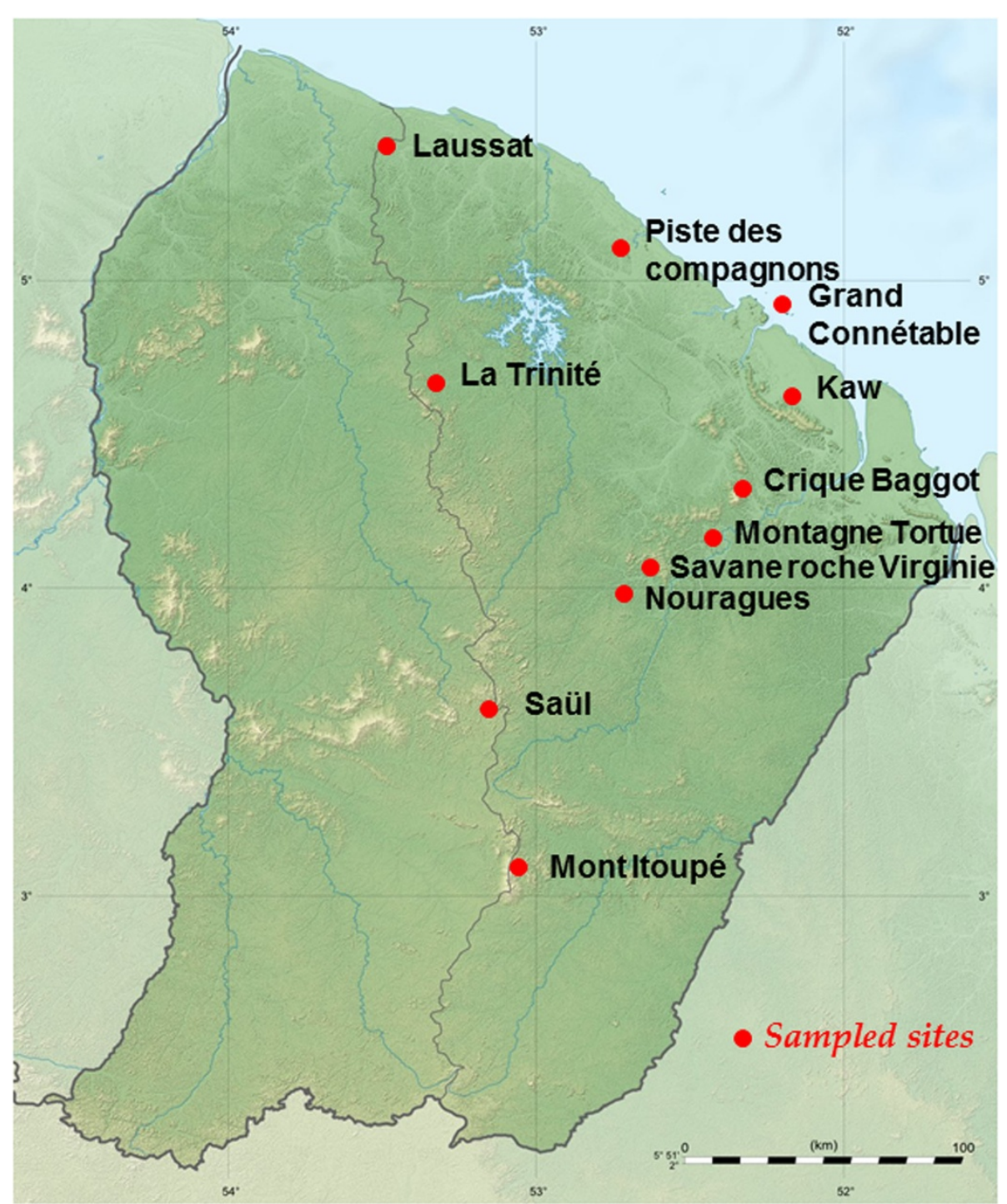

Figure 2 Map of French Guiana with the sampling sites.

each site) over a six months period (dry season). At the Mont Itoupe site, six window pane traps and six Malaise traps over one month (dry season) were used to collect spiders. At the Piste des compagnons site, two Malaise traps were placed over a two month period (dry season). Therefore, because of this non-standardization of sampling effort and methods, only a global estimation of total number of species was statistically feasible and no advanced biodiversity studies on spiders (Feest \& Cardoso 2012; Cardoso et al. 2009) were possible.

\section{Storage and identification}

Spiders were stored in labeled tubes containing 70\% ethanol. Material was identified first as Morphospecies (M-S) by the authors and when possible, identified at the species level by them. Otherwise, material was sent to family specialists (see Acknowledgments) for a complete identification or description. Juveniles were excluded from the list. Specimens are noted as "sp. n" only when recognized as a species new to science by a specialist of the family who will describe it in a further publication.

\section{Data analysis}

An accumulation curve plotting the number of collected individuals by the number of $\mathrm{M}-\mathrm{S}$ found was drawn to assess the species richness found in French Guiana. This accumulation curve was drawn with only seven of the sampling sites, because a quantitative protocol accompanied with photography of each specimen were applied only at those sites (Vedel and Lalagüe 2013). Although the number of sites (seven) for any statistical study is low, a rough estimation of the total number of spider 
species found can be nevertheless computed with a high standard deviation to consider. A logarithmic tendency curve (Colwell and Coddington 1994) (and its equation) was also drawn by Excel open office to derive the rate of the M-S number increase from our samplings (Table 4 and Figure 1).

In addition, to estimate the total species richness of French Guiana we computed, from our sampling, the two most widely used estimators: the Chao 1 (Chao 2005, Gotelli and Colwell 2010) was manually calculated, and the Jackknife computed online (http://www.mbrpwrc.usgs.gov/software/specrich.html) (Burnham and Overton 1979). Chao 1 is a minimum estimator of the species richness particularly adapted when the number of singletons and doubletons are high, and therefore well adapted to our case. Jackknife allows a non-biased estimation of the richness which is complementary of the first estimator used.

\section{Competing interests}

The authors declare that they have no competing interests.

\section{Authors' contributions}

W conducted the sampling and the analysis, updated the list and partly wrote the manuscript. CR gathered literature, identified Sparassidae specimens and participated at the manuscript writing. JM corrected the names and the references of the spider species list and partly wrote the manuscript. $A B$ updated the list and gathered literature, identified Anyphaenidae, Corinnidae and Ctenidae specimens and participated to the writing. All authors read and approved the final manuscript.

\section{Acknowledgements}

The first author would like to thank the SEAG (especially P-H. Dalens and S. Fernandez) for setting up most of the sampling trips contributing to this study, all the specialists which identified and provided specimens for this study (Courtial C., Edwards G.B., Gonzales A.B., Huber B., Lehtinen P.T., Lise A. A., Maréchal P., Santos A.J., Ysnel F.) and Greg Lamarre and Stéphane Brûlé for constructive discussions. We are indebted to Dr. Christopher David Williams who kindly corrected the English of this article. The authors thank four reviewers who improved substancially the manuscript with their comments. We also thank the following people for allowing and partly organizing the sampling trip : Maël Dewynter, ONF conservator of the natural reserve of Nouragues, Philippe Gaucher, Technical director of the Nouragues; Marguerite Delaval, ONF conservator of the natural reserve of la Trinité ; for the mission in Mount Itoupé, Jean-Claude Duss, director of Club Med ; Frédéric Mortier, director of Parc Amazonien de Guyane, Cécile Guitet chief of patrimoine naturel du Parc Amazonien and Nicolas Surugue, in charge of ecological mission. The two last authors thank the NPq (toADB) for financial support. This work has benefited from "Investissement d'Avenir" grants managed by Agence Nationale de la Recherche (CEBA, ref. ANR-10LABX-0025; TULIP, ref. ANR-10-LABX-41).

\footnotetext{
Author details

${ }^{1}$ Laboratoire d'entomologie Entobios, 5 Bis rue François Thomas, 97310 Kourou, Guyane Française, France. ${ }^{2}$ Laboratoire d'écologie intégrative, UMR ECOFOG, Université des Antilles et de la Guyane, Campus Agronomique de Kourou, 97310 Kourou, French Guiana, France. ${ }^{3}$ Laboratório Especial de Coleções Zoológicas, Instituto Butantan, Av. Vital Brasil, 1500, Butantã, São Paulo, SP, Brazil 05503-900. ${ }^{4}$ CNRS, EFA, UMR 5174 EDB (Laboratoire Evolution et Diversité Biologique), Université Paul Sabatier, 118 route de Narbonne, F-31062 Toulouse, France.
}

Received: 11 April 2013 Accepted: 18 July 2013

Published: 30 July 2013

\section{References}

Brescovit AD, De Oliveira U, Dos Santos AJ (2011) Aranhas (Araneae, Arachnida) do Estado de São Paulo, Brasil: diversidade, esforço amostral e estado do conhecimento. Biota Neotropica 11(1a):1-32

Burnham KP, Overton WS (1979) Robust estimation of population size when capture probabilities vary among animals. Ecology 60:927-936

Butchardt SHM, Walpole M, Collen B, van Strien A, Scharlemann JPW, Almond RAE, Baillie JEM, Bomhard B, Brown C, Bruno J, Carpenter KE, Carr GM, Chanson J, Chenery AM, Csirke J, Davidson NC, Dentener F, Foster M, Galli A, Glloway JN, Genovesi P, Gregory RD, Hockings M, Kapos V, Lamarque J-F, Leverington F, Loh J, McGeoch MA, McRae L, Minasyan A, et al. (2010) Global biodiversity: indicators of recent declines. Science 328:1164-1168

Cardoso P (2009) Standardization and optimization of arthropod inventories the case of Iberian spiders. Biodivers Conserv 18:3949-3962

Cardoso P, Scharf N, Gaspar C, Henriques SS, Carvalho R, Pedro HC, Schmidt JB, Silva I, Szüts T, de Castro A, Crespo LC (2008) Rapid biodiversity assessment (Araneae) using semi-quantitative sampling: a case-study in a Mediterranean forest. Insect conserv Div 1:71-84

Cardoso P, Pekar S, Jocqué R, Coddington JA (2011) Global Patterns of Guild Composition and Functional Diversity of Spiders. PLoS One 6(6):e21710

Chao A (2005) Species richness estimation. In: Balakrishnan N, Read CB, Vidakovic B (ed) Encyclopedia of Statistical Sciences. Wiley, New York, pp 7909-7916

Coddington JA, Young LH, Coyle FA (1996) Estimating spider species richness in a southern Appalachian cove hardwood forest. J Arachnol 24:111-128

Coddington JA, Agnarsson I, Miler JA, Kuntner M, Hormiga G (2009) Undersampling bias: the null hypothesis for singleton species in tropical arthropod surveys. J Anim Ecol 78:573-584

Colwell RK, Coddington JA (1994) Estimating terrestrial biodiversity through extrapolation. Phil Trans R Soc Lond B 345:101-118

De Baan L, Alkemade R, Koellner T (2012) Land use impacts on biodiversity in LCA: a global approach. Int J Life Cycle Assess. http://dx.doi.org/10.1007/ s11367-012-0412-0

Di Caporiacco L (1954) Araignées de la Guyane Française du Muséum d'Histoire Naturelle de Paris. Commentationes Pontificiae Academiae Scientiarum 16:45-193

Dias SC, Carvalho LS, Bonaldo AB, Brescovit AD (2010) Refining the establishment of guilds in Neotropical spiders (Arachnida: Araneae). J Nat Hist 44(3-4):219-239

Drolshagen B, Bäckstam CM (2011) Notes on the genus Harmonicon F.O.PCambridge, 1896 (Araneae, Dipluridae) with description of a new species from French Guyana. ZooKeys 112:89-96

EEA (European Environment Agency (2005) The European Environment - State andOutlook 2005. European Environment Agency, EEA Report 4/2009. Progress Towards the European 2010 Biodiversity Target. EEA Copenhagen, Denmark, Copenhagen, p 52

Feest A (2013) The utility of the Streamlining European Biodiversity Indicators 2010 (SEBI 2010). ECol Indic 28:16-21

Feest A, Cardoso P (2012) The comparison of site spider "biodiversity quality" in Portuguese protected areas. Ecol Indic 14:229-235

Feest A, van Swaay C, Aldred TD, Jedamzik K (2011) The biodiversity quality of butterfly sites: a metadata assessment. Ecol Indic 11:669-675

Floren A, Deeleman-Reinhold C (2005) Diversity of arboreal spiders in primary and disturbed tropical forests. J Arachnol 33:323-333

Foelix RF (1996) Biology of Spiders. Oxford University Press, New York. 432pp

Gotelli NJ, Colwell RK (2010) Estimating species richness. In: Magurran AE, McGill BJ (ed) Biological diversity: Frontiers in measurement and assessment. Oxford, UK, Oxford University Press

Jones JPG, Collen B, Atkinson G, Baxter PWJ, Bubb P, Illian JB, Katzner TE, Keane A, Loh J, McDonald-Madden E, Nicholson E, Pereira HM, Possingham HP, Pullin AS, Rodrigues ASL, Ruiz-Gutierrez V, Sommerville M, Millner-Gulland EJ (2011) The why, what and how of global biodiversity indicators beyond the 2010 target. Conserv Biol 25(3):450-457

Lopez A (1988) Araignées de Guyane Française. La sous-famille des Gasteracanthinae O. Pickard-Cambridge (Araneidae) et les proies de Sceliphron fistalarium (Dahlbom) (Hymenoptera : Specidae). Sciences Nat 82:14-22

New TR (1999) Untangling the web: spiders and the challenges of invertebrate conservation. J of Ins Cons 3:251-256

Pinkus-Rendón MA, León-Cortés JL, Ibarra-Núñez G (2006) Spider diversity in a tropical habitat gradient in Chiapas. Mexico Divers Distrib 12:61-69

Platnick NI (2012) The world spider catalog, version 13.0. American Museum of Natural History online. http://research.amnh.org/iz/spiders/catalog"http:// research.amnh.org/iz/spiders/catalog (assessed in: 19.VIII.2012) 
Prószyński J (2012) Monograph of the Salticidae (Araneae) of the World 1995 2012. http://www.peckhamia.com/salticidae/salticidae.php (assessed in: 19. VIII.2012)

Scholes RJ, Mace GM, Turner W, Geller GN, Jurgens N, Larigauderie A, Muchoney D, Wather BA, Mooney HA (2008) Ecology: toward a global bio-diversity observing system. Science 321:1044-1045

Sørensen LL, Coddington JA, Scharff N (2002) Inventorying and estimating subcanopy spider diversity using semi-quantitative sampling methods in an Afromontane forest. Environ Entomol 31:319-330

Targetti S, Viaggi D, Cuming D, Sarthou JP, Choisis JP (2012) Assessing the costs of measuring biodiversity: methodological and empirical issues. Food Economics 9(1-2):2-9

Vedel V, Lalagüe H (2013) Standardized sampling protocol for spider community assessment in the neotropical rainforest. J of Ent Zoo Stud 1(2):18-34

Vedel V, Camus D, Lamarre G (2011) Malaise and glass traps: useful means of catching canopy-dwelling spiders? Newsl Brit. Arachnol Soc 122:12-15

doi:10.1186/2193-1801-2-361

Cite this article as: Vedel et al:: Biodiversity baseline of the French Guiana spider fauna. SpringerPlus 2013 2:361.

\section{Submit your manuscript to a SpringerOpen ${ }^{\circ}$} journal and benefit from:

- Convenient online submission

- Rigorous peer review

- Immediate publication on acceptance

- Open access: articles freely available online

- High visibility within the field

- Retaining the copyright to your article 